\title{
Single tumor imaging with multiple antibodies targeting different antigens
}

Yujing Sun ${ }^{1}$, Girja Shukla ${ }^{1}$, Stephanie C. Pero', Erika Currier ${ }^{2}$, Giselle Sholler ${ }^{2}$, and David Krag ${ }^{1}$

${ }^{1}$ Department of Surgery and ${ }^{2}$ Department of Pediatrics, Vermont Cancer Center, University of Vermont College of Medicine, Burlington, VT, USA

Antibodies are important drugs for treating cancer and there is strong rationale for using multiple antibodies to improve outcomes. We labeled two breast cancer binding antibodies, anti-ErbB2 and anti-EpCAM, with infrared fluorescence dyes of different wavelengths and determined their in vivo distribution in a breast cancer xenograft model using a near-infrared (NIR) fluorescence imaging system. Our data show that these two antibodies can be readily assessed simultaneously in mouse xenograft model. This will help guide design of dosing strategies for multiple antibodies and identify potential interaction that could affect pharmacokinetics and possible side effects.

BioTechniques 52:1-3 (April 2012) doi 10.2144/000113855

Keywords: combination administration; antibody; near-infrared fluorescence imaging; breast cancer; xenograft; mouse

Tumor-targeting antibodies can inhibit tumor cell proliferation by inhibition of signaling receptors on tumor cell membrane (1), evoking host anti-tumor immune response $(2,3)$, or delivering cytotoxic drugs (4). The therapeutic effect of antibodies can be increased by simultaneous administration of antibodies with complementary action or asymmetric side effect profiles (5-8).

Accumulation of antibodies in tumor and normal tissue is variable, complex, and unpredictable. Near-infrared (NIR) imaging allows extended imaging of fluorescently labeled antibodies in small animal models. Tumor uptake studies have been done with a variety of individual NIR fluorophorelabeled drugs $(9,10)$ but limited data are available for tracking multiple antibodies simultaneously (7,11-15).

We report here NIR simultaneous imaging of two different tumor-targeting antibodies in a mouse xenograft model. This allowed tumor and normal tissue uptake evaluation of two antibodies simultaneously over an extended period of time. This will help guide design of dosing strategies and identify potential interaction of the antibodies that could affect improved as well as adverse outcomes.

The NIR dyes XenoLight 680 succinimidyl ester (SEM) and 770 SEM (Caliper LifeSciences, Hopkinton, MA, USA) were used tolabelHER2/neu antibody (Herceptin, Genentech, South San Francisco, CA, USA) and anti-human EpCAM monoclonal antibody (R\&D Systems, Minneapolis, MN, USA). In brief, $1 \mathrm{~mL} 2.5 \mathrm{mg} / \mathrm{mL}$ antibody solution in $0.1 \mathrm{M}$ sodium bicarbonate buffer $(\mathrm{pH} \sim 8.3)$ was prepared, and 0.25 umol Xenolight CF770 SEM or CF680 SEM was added in a drop-wise fashion while stirring the reaction solution. The reaction was performed at room temperature $2 \mathrm{~h}$ in dark while rocking. After the reaction, labeled antibodies were separated from free dye and concentrated using centrifuge filter devices with nominal molecular weight limit at 10,000 (cat no. UFC901024, Amicon Ultra; Millipore, Bedford, MA, USA). The degree of labeling was measured based on the following formula: [conjugate] (mg/ $\mathrm{mL})=\left\{\left[\mathrm{A}_{280}-\left(\mathrm{A}_{\max } \times \mathrm{CF}\right)\right] / 1.4\right\} \times$ dilution factor ( $\mathrm{CF}$ is the absorbance correction factor: 0.06 for XenoLight CF770 and 0.09 for XenoLight CF680). The conjugated antibodies were stored at $4^{\circ} \mathrm{C}$ and used for animal injection within 1 week.

Mouse BT474 xenograft generation and all experiments were performed according to a protocol approved by Institutional Animal Care and Use Committee (IACUC) at the University of Vermont. Antibody uptake studies were done when the flank xenografts reached 0.4 to $0.6 \mathrm{~cm}$.

Groups of mice were injected intravenously with a bolus of one or both of 1 nmol Herceptin-680 or anti-EpCAM-770. Control groups were injected with the same amount of correspondingly labeled mouse or human irrelevant IgG. Dye control groups received equimolar quantities of the free dyes. Images were obtained $30 \mathrm{~min}$ postinjection and then daily for 10 days (IVIS Lumina; Caliper Life Sciences). Uptake was referenced to the tumor and the kidneys.

We compared the uptake of a single antibody injection per mouse for either Herceptin-680 or anti-EpCAM-770 and compared that to control antibody and free dye (Figure 1). Preferential accumulation of both Herceptin- 680 and anti-EpCAM-770 was observed in the BT474 xenograft up to 96 h. Both Herceptin-680 and anti-

\section{Method summary:}

Here we provide a methodology for simultaneously imaging two antibodies labeled with infrared fluorescent dyes of differing wavelengths in a single tumor using near infrared (NIR) fluorescence imaging. This approach enables assessment of multiple antibody accumulation in a single tumor and normal body tissues. 
EpCAM-770 had higher concentrations in tumor tissues at all time points, but some accumulation of anti-EpCAM-770 was observed also in the kidneys. Studies were not done to confirm whether the full antiEpCAM-770 antibody or cleaved conjugates were present in the kidney.

Direct comparison was made with double antibody versus single antibody injection. Three mice were used for each experiment. One mouse received Herceptin-680 alone, one mouse received both Herceptin-680 and anti-EpCAM-770 antibodies, and one mouse received anti-EpCAM-770 alone (Figure 2). Herceptin and anti-EpCAM antibodies accumulated preferentially in the BT474 tumors when injected as a single antibody and also accumulated preferentially in the tumors when injected simultaneously. Figure $2 \mathrm{~B}$ shows tumor accumulation of Herceptin when injected as the only antibody or when coinjected with the antiEpCAM antibody. Figure 2C shows antiEpCAM accumulation under the same conditions. Both antibodies had the same pattern when administered either individually or simultaneously.

Antibodies are an important category of anticancer drugs, and a large number of antibodies are in preclinical and clinical development. Simultaneous administration of multiple antibodies that target different antigens or epitopes may be a successful strategy for increasing anticancer action and overcoming resistance. Simultaneous treatment with multiple antibodies promotes tumor inhibition by blocking different cellular functions in the same cell. Also, the antigenic profile varies from cell to cell within a tumor. Treatment with a single antibody will be less effective on low-antigen cells. Multiple antibodies targeting different antigens may offset the problem of variable antigen levels in a tumor.

Antibody accumulation in a solid tumor is complex, and variables such as the binding site barrier may impact accumulation. Other variables include speed of internalization, affinity, and rate of degradation. Antibodies targeting the same epitope with different binding properties have variable accumulation in a solid cancer (16). For example, in vitro binding affinity did not predict in vivo xenograft accumulation of multiple anti-melanoma antibodies (17). Preclinical experiments with in vivo imaging of multiple antibodies will be important to identify dosing strategies and to assess the impact of antibody variables and tumor variables on tumor uptake.

NIR imaging has previously been used to evaluate interaction of two antibodies administered simultaneously. This has been done by labeling one antibody at a

Filter set (Exc/Emi 675/cy5.5) Filter set (Exc/Emi 745/ICG)

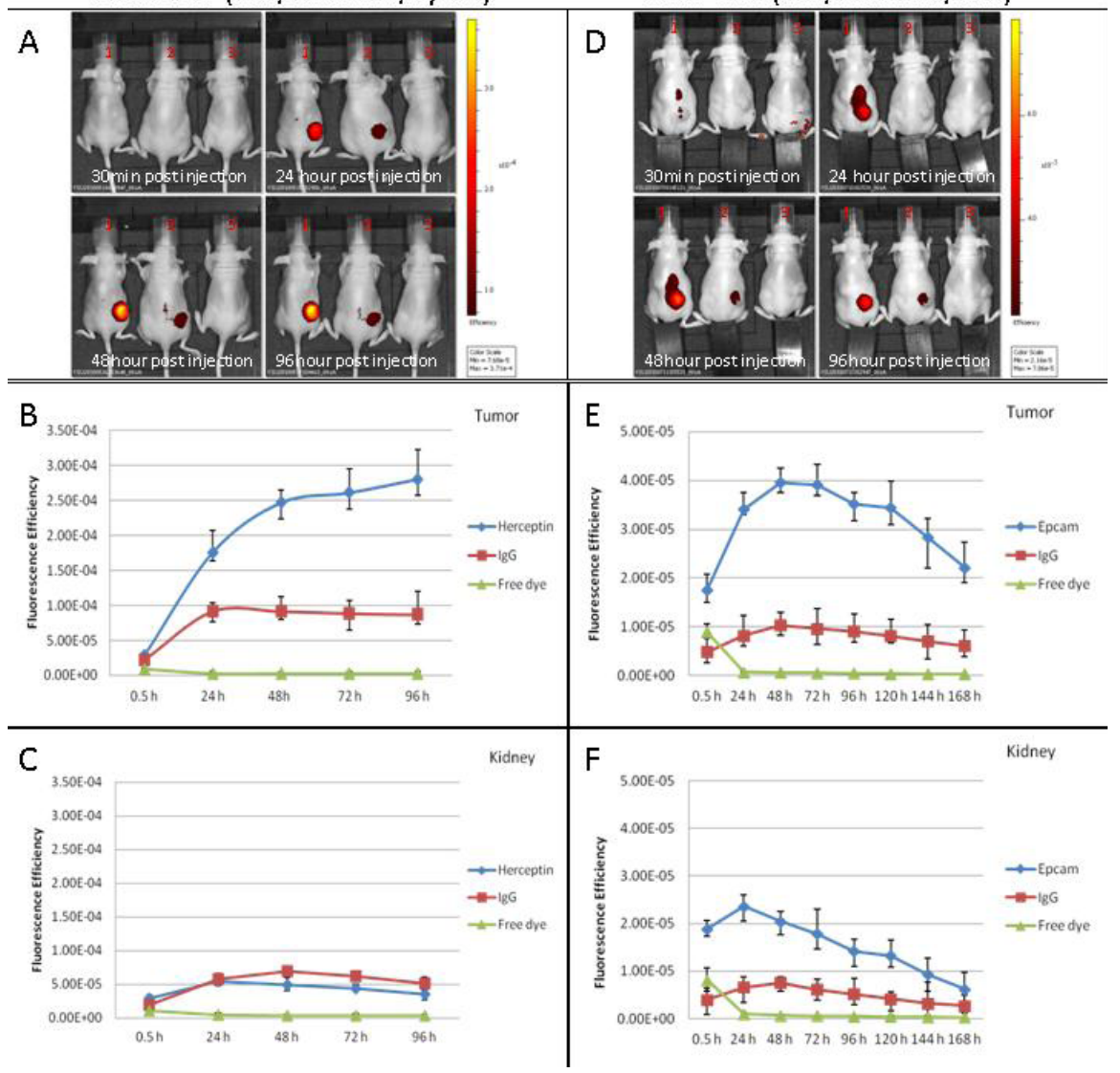

Figure 1. Representative images and signal quantification over time ( $30 \mathrm{~min}$ to $96 \mathrm{~h}$ ) for infusion of a single antibody in two groups of mice. Each test group had three mice. The \#1 mouse in each group was injected with test antibody, the \#2 mouse in the middle was injected with irrelevant labeled antibody, and the \#3 mouse on the right was injected with free dye. The first column (images A, B, C) shows results for Herceptin-680 and the second column (images D, E, F) show results for antiEpCAM-770. The images show preferential accumulation of Herceptin and anti-EpCAM antibody in the tumor. Also, the anti-EpCAM antibody had some uptake in the kidney while Herceptin (C) had no specific kidney uptake.

time and assessing the alteration of uptake when the second unlabeled antibody was administered (7). Dual imaging, as we report here, would have allowed simultaneous assessment of both antibodies in vivo. Multiple NIR-labeled antibodies have also been used to image multiple tumors of different histology implanted simultaneously in mice. For example, following intraperitoneal injection of NIR antibodies, surgically exposed peritoneal xenografts with either one or two different tumors were imaged at a single point in time in euthanized mice $(12,15)$. Other studies were designed to diagnose tumors of different histology. Here multiple tumors with different histology were implanted in different locations and imaged following intravenous injection of NIR antibodies specific for each tumor type $(13,14)$. In our study, one tumor type was implanted and visualized with two NIR antibodies specific for the one tumor but targeting different antigens. Our goal was to evaluate NIR-labeled antibodies to simulate cancer treatments of a single tumor using multiple antibodies. Our work demonstrates that this approach is feasible for assessing pharmacokinetics and potential interactions of multiple antibodies targeting a single tumor.

Simultaneous administration of multiple antitumor antibodies may result in increased adverse events. This may be especially important for antibodies that are conjugated with cytotoxic drugs. For example, both antibodies may share undesirable cross-binding to the same noncancer tissue and together lead to increased normal tissue damage. Also, common metabolic processing of the antibodies may lead to unexpected accumulation in the liver or kidney, resulting in unanticipated adverse events. Given the potential variation of tumor and normal tissue accumulation of multiple antibodies, simultaneous NIR imaging of multiple antibodies will be valuable. We observed that the normal tissue accumulation profile was different between the two antibodies we tested; Herceptin had 


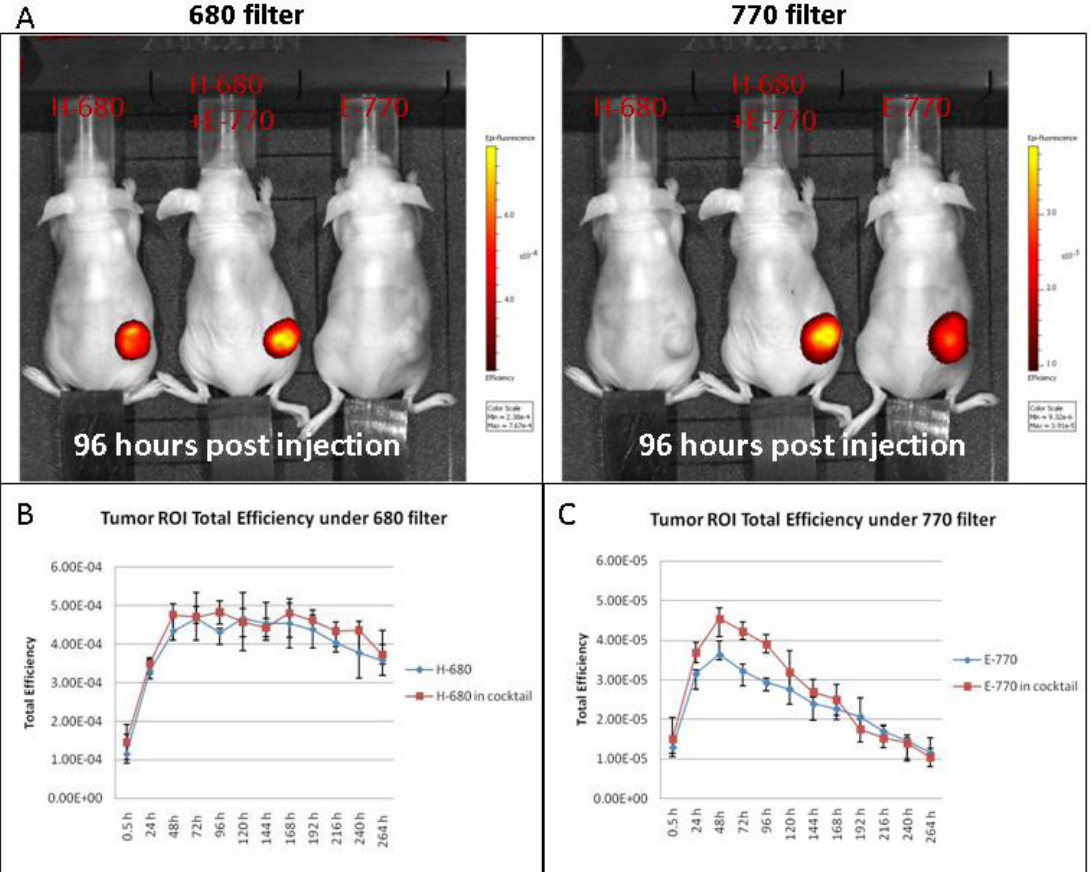

Figure 2. Images (at $96 \mathrm{~h}$ ) and signal quantification over time (30 $\mathrm{min}$ to $264 \mathrm{~h}$ ) for one representative group of mice infused with two antibodies simultaneously compared with a single antibody. This group of three mice was imaged twice in rapid sequence at each time point. The image on the left was generated using the 680 filter set for selective imaging of the Herceptin antibody and the image on the right was generated using the $\mathbf{7 7 0}$ filter set for selective imaging of the anti-EpCAM antibody. In this group of three mice, the mouse on the left was injected with Herceptin-680 only, the middle mouse received both antibodies, and the mouse on the right received anti-EpCAM-770 only. Graph B shows tumor accumulation of Herceptin when injected as the only antibody or when co-injected with the anti-EpCAM antibody. Graph C shows anti-EpCAM accumulation under the same conditions. Both antibodies had same pattern when administered either individually or simultaneously.

minimal uptake in normal organs, whereas the anti-EpCAM antibody had accumulation in the liver and kidney.

In conclusion, administration of multiple antibodies is complex, and our study demonstrated that two antibodies labeled with different NIR dyes allowed simultaneous assessment of antibody accumulation in a single tumor and normal body tissues. In vivo assessment of tumor and normal tissue pharmacokinetics for multiple antibodies simultaneously will help guide design of dosing strategies and identify potential interaction of the antibodies that could affect pharmacokinetics.

\section{Acknowledgments}

This study was supported by the Department of Defense Breast Cancer Research Program (BCRP) BC087174 and in part by SD Ireland Professorship of Oncology for Research, Andrew's Fund, and the Meryl \& Charles Witmer Charitable Foundation.

\section{Competing interests}

The authors declare no competing interests.

\section{References}

1. Huang, Z.Q. and D.J. Buchsbaum. 2009. Monoclonal antibodies in the treatment of pancreatic cancer. Immunotherapy 1:223-229.

2. Saxena, R. and A. Dwivedi. 2010. ErbB family receptor inhibitors as therapeutic agents in breast cancer: current status and future clinical perspective. Med. Res. Rev. Oct 25. [Epub ahead of print]

3. Ranieri, G., R. Patruno, E. Ruggieri, S. Montemurro, P. Valerio, and D. Ribatti. 2006. Vascular endothelial growth factor (VEGF) as a target of bevacizumab in cancer: from the biology to the clinic. Curr. Med. Chem. 13:18451857.

4. McCarron, P.A., S.A. Olwill, W.M. Marouf, R.J. Buick, B. Walker, and C.J. Scott. 2005. Antibody conjugates and therapeutic strategies. Mol. Interv. 5:368-380.

5. Friess, T., W. Scheuer, and M. Hasmann. 2005. Combination treatment with erlotinib and pertuzumab against human tumor xenografts is superior to monotherapy. Clin. Cancer Res. 11:5300-5309.

6. Marini, P., S. Denzinger, D. Schiller, S. Kauder, S. Welz, R. Humphreys, P.T. Daniel, V. Jendrossek, et al. 2006. Combined treatment of colorectal tumours with agonistic TRAIL receptor antibodies HGS-ETR1 and HGS-ETR2 and radiotherapy: enhanced effects in vitro and dose-dependent growth delay in vivo. Oncogene 25:5145-5154.

7. Scheuer, W., T. Friess, H. Burtscher, B. Bossenmaier, J. Endl, and M. Hasmann. 2009. Strongly enhanced antitumor activity of trastuzumab and pertuzumab combination treatment on HER2-positive human xenograft tumor models2009; No. 24:9330-9336. Located at: Cancer Res 24:9330-9336.

8. Milenic, D.E., E.D. Brady, K. Garmestani, P.S. Albert, A. Abdulla, and M.W. Brechbiel. 2010. Improved efficacy of alpha-particletargeted radiation therapy: dual targeting of human epidermal growth factor receptor-2 and tumor-associated glycoprotein 72 . Cancer 116(Suppl):1059-1066.

9. Wang, H., K. Chen, G. Niu, and X. Chen. 2009. Site-specifically biotinylated VEGF(121) for near-infrared fluorescence imaging of tumor angiogenesis. Mol. Pharm. 6:285-294.

10. Manning, H.C., N.B. Merchant, A.C. Foutch, J.M. Virostko, S.K. Wyatt, C. Shah, E.T McKinley,J.Xie, et al. 2008. Molecular imaging of therapeutic response to epidermal growth factor receptor blockade in colorectal cancer. Clin. Cancer Res. 14:7413-7422.

11. Gong, H., J. Kovar, G. Little, H. Chen, and D.M. Olive. 2010. In vivo imaging of xenograft tumors using an epidermal growth factor receptor-specific affibody molecule labeled with a near-infrared fluorophore. Neoplasia 12:139-149.

12. Longmire, M., N. Kosaka, M. Ogawa, P.L. Choyke, and H. Kobayashi. 2009. Multicolor in vivo targeted imaging to guide real-time surgery of HER2-positive micrometastases in a two-tumor coincident model of ovarian cancer. Cancer Sci. 100:1099-1104.

13. Koyama, Y., T. Barrett, Y. Hama, G. Ravizzini, P.L. Choyke, and H. Kobayashi. 2007. In vivo molecular imaging to diagnose and subtype tumors through receptor-targeted optically labeled monoclonal antibodies. Neoplasia 9:1021-1029.

14. Barrett, T., Y. Koyama, Y. Hama, G. Ravizzini, I.S. Shin, B.S. Jang, C.H. Paik, Y. Urano, et al. 2007. In vivo diagnosis of epidermal growth factor receptor expression using molecular imaging with a cocktail of optically labeled monoclonal antibodies. Clin. Cancer Res. 13:6639-6648

15. Kosaka, N., M. Ogawa, M.R. Longmire, P.L. Choyke, and H. Kobayashi. 2009. Multitargeted multi-color in vivo optical imaging in a model of disseminated peritoneal ovarian cancer. J. Biomed. Opt. 14:014023.

16. Rudnick, S.I., J. Lou, C.C. Shaller, Y. Tang, A.J. Klein-Szanto, L.M. Weiner, J.D. Marks, and G.P. Adams. 2011. Influence of affinity and antigen internalization on the uptake and penetration of anti-HER 2 antibodies in solid tumors. Cancer Res. 71:2250-2259.

17. McCready, D.R., C.M. Balch, I.J. Fidler, and J.L. Murray. 1989. Lack of comparability between binding of monoclonal antibodies to melanoma cells in vitro and localization in vivo. J. Natl. Cancer Inst. 81:682-687.

Received 14 December 2011; accepted 20 February 2012.

Address correspondence to David Krag, Department of Surgery, Vermont Cancer Center, University of Vermont College of Medicine, Burlington, VT, USA. Email address: david. krag@uvm.edu

To purchase reprints of this article, contact: biotechniques@fosterprinting.com 